\title{
Large-scale roll-to-roll printed, flexible and stable organic bulk heterojunction photodetector
}

Sichao Tong ${ }^{1}$, Jun Yuan ${ }^{2}$, Chujun Zhang ${ }^{1}$, Chunhua Wang ${ }^{1}$, Baoxing Liu ${ }^{1}$, Jianqiang Shen ${ }^{1}$, Huayan Xia ${ }^{1}$, Yingping Zou ${ }^{2}$, Haipeng Xie ${ }^{1}$, Jia Sun ${ }^{1}$, Si Xiao ${ }^{1}$, Jun $\mathrm{He}^{1}$, Yongli Gao ${ }^{1,3}$ and Junliang Yang ${ }^{1}$

A flexible and stable photodetector shows great potential applications in intelligent wearable devices, health monitoring, and biological sensing. The high-output fabrication of flexible and stable photodetector via the large-scale printing process would accelerate its commercialization. Herein, a high performance, flexible organic bulk heterojunction (BHJ) photodetector with good stability is designed and fabricated via a large-scale roll-to-roll (R2R) micro-gravure printing technique on polyethylene terephthalate (PET) or paper substrate, in which the organic BHJ active layer is structured with $[6,6]$-phenyl $\mathrm{C}_{61}$ butyric acid methyl ester (PCBM) and a donor-acceptor copolymer, i.e., employing 4,8-bis(2-ethylhexylthiophene) benzo[1,2-b;3,4-b'] dithiophene $(\mathrm{BDTT})$ as the donor unit and 5,8-bis(5-thiophen-2-yl)-6,7-difluoro-2,3-bis(4-ethylhexyloxy-1-mata-luorophenyl) quinoxaline (ffQx) as the acceptor unit (PBDTT-ffQx). The PBDTT-ffQx/PCBM BHJ photodetector shows a broad photoresponse in ultraviolet and visible light, a high detectivity $\left(D^{*}\right)$ value up to $6.19 \times 10^{11}$ Jones, and an excellent $I_{\text {photo }} / I_{\text {dark }}$ as high as $5.6 \times 10^{2}$. It exhibits excellent flexibility and stability. Its performance parameters could maintain over $80 \%$ of original values after bending 10,000 cycles or exposing in ambient condition (humidity $\sim 50 \%$, temperature $\sim 30^{\circ} \mathrm{C}$ ) for 50 days without any encapsulation. More importantly, the R2R micro-gravure printed PBDTT-ffQx/PCBM BHJ active layer is great homogeneous, and the responsivity $(R)$ values of photodetector arrays show a very narrow distribution. The research results show that a high-performance PBDTT-ffQx/PCBM BHJ photodetector with well reliability and reproducibility can be fabricated via the R2R micro-gravure printing technique, which provides an available strategy for fabricating large-area and flexible electronic and optoelectronic devices.

npj Flexible Electronics (2018)2:7; doi:10.1038/s41528-017-0020-y

\section{INTRODUCTION}

The photodetector is an important optoelectronic device, and directly converts the incident light to electrical signal. ${ }^{1-4}$ Organic photodetector has been attracting great attention and shows widely potential applications in intelligent wearable devices, health monitoring, biological sensing, and so on, resulting from the advantages of low cost, solution-processability, flexibility, as well as chemically tailoring and structural diversity for organic semiconductor materials. ${ }^{5-7}$ With the rapid development of chemical designs, all kinds of novel organic semiconductors with customized properties have been synthesized for optoelectronic devices, ${ }^{8-10}$ and some conjugated polymers have been employed as the active materials in an organic photodetector, such as monoaniline-capped poly[(4,4'-bis(2-ethylhexyl)-dithieno[3,2-b:2', $\left.3^{\prime}-d\right]$ silole)-2,6-diyl-alt-(2,1,3-enzothiadiazole)-4,7-diyl] $\quad\left(\right.$ PSBTBT- $\left.\mathrm{NH}_{2}\right)$ and a copolymer of 4,4-bis(2-ethylhexyl)-dithieno[3,2-b:2', $\left.3^{\prime}-d\right]$ silole and $n$-octylthieno[3,4-c]pyrrole-4,6-dione (PDTSTPD). ${ }^{11-13}$ Blending the active materials to form bulk heterojunction (BHJ) would greatly improve certain performance parameters of the organic photodetector, implying that $\mathrm{BHJ}$ is a possible route to enhance the performance of the organic photodetector. ${ }^{14}$

Roll-to-roll (R2R) printing techniques, offering the advantages of large-scale and low-cost process and high output with highquality printed films, have been developed to fabricate large-area organic solar cells and a perovskite nanowire photodetector. ${ }^{15-17}$ However, almost all of high-performance organic optoelectronic devices are fabricated by spin-coating or thermal evaporation techniques in laboratory, which are high-material-wasting and low-output fabrication process. ${ }^{18,19}$ Thus, it is very meaningful to further study R2R printing parameters and technologies for producing high-performance optoelectronic devices, which would accelerate the commercialization of printed optoelectronic devices.

Our newly developed organic donor-acceptor (D-A)-conjugated polymer material, employing 4,8-bis(2-ethylhexylthiophene) benzo[1,2-b;3,4- $\left.b^{\prime}\right]$ dithiophene (BDTT) as the donor unit and 5,8bis(5-thiophen-2-yl)-6,7-difluoro-2,3-bis(4-ethylhexyloxy-1-mataluorophenyl) quinoxaline (ffQx) as the acceptor unit (PBDTT-ffQx), was used in polymer solar cells and exhibited a power conversion efficiency of $8.6 \%$, showing excellent light absorption properties. ${ }^{20}$ Herein, the high-performance, flexible organic photodetector is designed with bulk heterjunction (BHJ) composed of PBDTT-ffQx and $[6,6]$-Phenyl $C_{61}$ butyric acid methyl ester (PCBM), and fabricated by the large-scale, R2R micro-gravure printing technique on polyethylene terephthalate (PET) and paper substrate. The PBDTT-ffQx/PCBM BHJ photodetector shows a broad photoresponse in ultraviolet (UV) and visible (Vis) light with responsivity $(R)$ over $0.92 \mathrm{~A} / \mathrm{W}$, a high detectivity $\left(D^{*}\right)$ value up to $6.19 \times 10^{11}$

\footnotetext{
${ }^{1}$ Hunan Key Laboratory for Super-Microstructure and Ultrafast Process, School of Physics and Electronics, Central South University, Changsha 410083 Hunan, China; ${ }^{2}$ College of Chemistry and Chemical Engineering, Central South University, Changsha 410083 Hunan, China and ${ }^{3}$ Department of Physics and Astronomy, University of Rochester, Rochester, NY 14627, USA

Correspondence: Junliang Yang (junliang.yang@csu.edu.cn)
}

Received: 8 October 2017 Revised: 8 December 2017 Accepted: 12 December 2017

Published online: 01 March 2018 
(a)
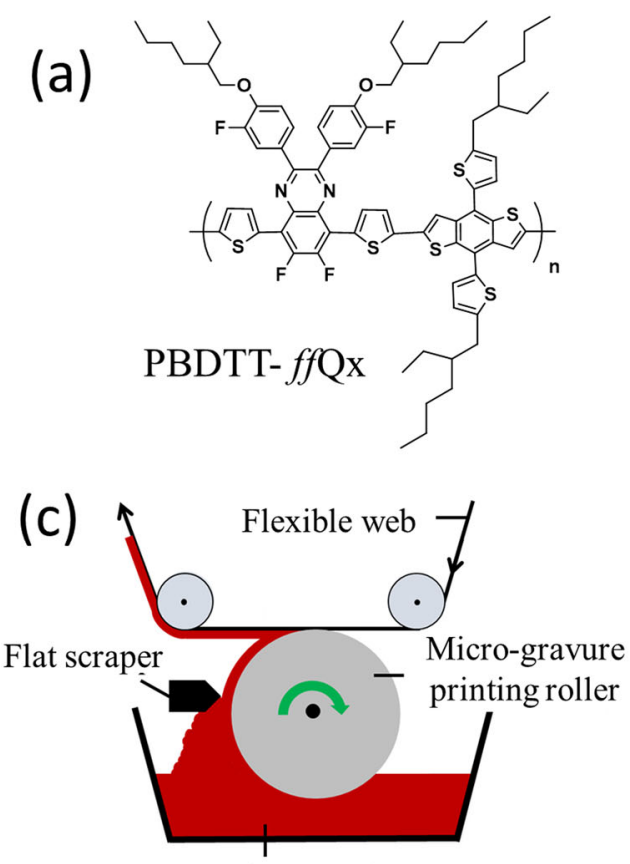

(b)

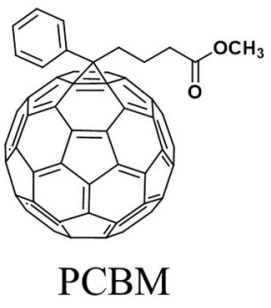

(d)

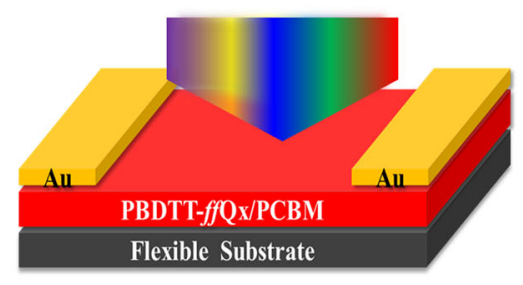

Ink reservoir

Fig. 1 Chemical structures of a PBDTT-ffQx and b PCBM. c Schematic of micro-gravure printing process. d Schematic configuration of the PBDTT-ffQx/PCBM BHJ photodetector

Jones, and an excellent $I_{\text {photo }} / I_{\text {dark }}$ as high as $5.6 \times 10^{2}$. More importantly, the distribution of $R$ values is in a very small scope for the R2R printed, large-area photodetector arrays, implying that $\mathrm{R} 2 \mathrm{R}$ printed $\mathrm{BHJ}$ thin film is very homogeneous with good reliability and reproducibility. Meanwhile, the performance parameters can maintain over $80 \%$ of original values after bending 10,000 cycles or exposing in ambient condition for 50 days without any encapsulation.

\section{RESULTS AND DISCUSSION}

R2R micro-gravure printed PBDTT-ffQx/PCBM BHJ thin film

The molecular structure of conjugated polymer PBDTT-ffQx is shown in Fig. 1a, which is constituted with a donor unit of BDTT and an acceptor unit of ffQx. The PBDTT-ffQx and PCBM (Fig. 1b) were blended to form the active materials. The PBDTT-ffQx/PCBM $\mathrm{BHJ}$ active thin film was fabricated by a large-scale, R2R microgravure printing method (Fig. 1c), and the schematic configuration of PBDTT-ffQx/PCBM BHJ photodetector is shown in Fig. 1d. The R2R micro-gravure printed, large-area PBDTT-ffQx/PCBM BHJ thin films on flexible PET and paper are shown in Figure S1. Because of long branched chain structure of PBDTT-ffQx, the photongenerated electrons could be transferred inside the molecular and adjacent molecules with low recombination. ${ }^{20}$ Meanwhile, the PCBM could play as a significant role for extracting and transferring photon-generated carriers. ${ }^{21}$ The PBDTT-ffQx and PCBM are excellent organic semiconductor materials with good air stability and flexibility, as well as energy level matching with each other, which would lead to great potentials in high-performance organic photodetectors.

The surface morphology of PBDTT-ffQx/PCBM BHJ thin film is shown in Fig. $2 \mathrm{a}$ and $\mathrm{b}$. With optimizing the printing parameters, the PBDTT-ffQx/PCBM BHJ thin film shows a smooth and compact morphology with the surface root-mean-square roughness as low as $5.64 \mathrm{~nm}$, which would cause to a low density of interfacial defects. $^{22}$ The scanning electron microscope (SEM) and optical microscopy morphology images of PBDTT-ffQx/PCBM BHJ thin film on PET in Figure S2a and c suggest that R2R micro-gravure printed thin film is smooth as well, but the rough paper substrate would result in a rough surface for PBDTT-ffQx/PCBM BHJ thin film (Figure S2b and d). The transmission electron microscope (TEM) image (Fig. 2b) indicates the microcrystal phase separation of PBDTT-ffQx/PCBM BHJ thin film, which is beneficial for charge transport in PBDTT-ffQx that could be separated at the interface between PBDTT-ffQx and PCBM. ${ }^{20}$ The very thin PBDTT-ffQx/PCBM BHJ film would not absorb sufficient light for producing electron-hole (e-h) pairs, while the very thick layer would lead to the recombination of electron-hole pairs. Thus, the optimized thickness of R2R printed PBDTT-ffQx/PCBM BHJ thin film is about $250 \mathrm{~nm}$, as the cross-sectional SEM image shown in Figure S3. Figure 2c shows the UV-Vis absorption spectra of PBDTT-ffQx, PCBM, and PBDTT-ffQx/PCBM BHJ thin films. For the PBDTT-ffQx thin film, there is strong and broad absorption ranged from 320 to $700 \mathrm{~nm}$, revealing that the PBDTT-ffQx is an excellent light harvester. While the main absorption peak of PCBM thin film is about $350 \mathrm{~nm}$. It is worth noting that the absorption spectra of PBDTT-ffQx/PCBM BHJ thin film includes both of the characteristic peaks of PBDTT-ffQx and PCBM, leading to the enhanced intensity of light absorption, which is potential for the high-performance photodetector. The time-resolved photoluminescence $(\mathrm{PL})$ traces are representative of the transient evolution of $\mathrm{e}-\mathrm{h}$ population after impulsive photoexcitation, which could be used to quantify the carrier dynamics and evaluate the carriers lifetime. ${ }^{23}$ The carrier lifetime of PBDTT-ffQx/PCBM BHJ thin film $(0.40 \mathrm{~ns})$ is almost double to that of PBDTT-ffQx thin film $(0.21 \mathrm{~ns})$, as shown in Fig. 2d. The PCMB is a classic electron transport material, but it could also have the ability of transporting hole. It has been proved that the fullerene materials could be acted as $p$-type layer in heterojunction organic solar cells with proper energy levels. ${ }^{24,25}$ Thus, the PCBM would extract the holes from the PBDTT-ffQx, leading to the decreased recombination of e- $h$ pairs in PBDTT$f f Q x$ thin film and the dramatically enhanced carrier lifetime, which would be helpful to produce the high-performance photodetector. $^{26}$ 

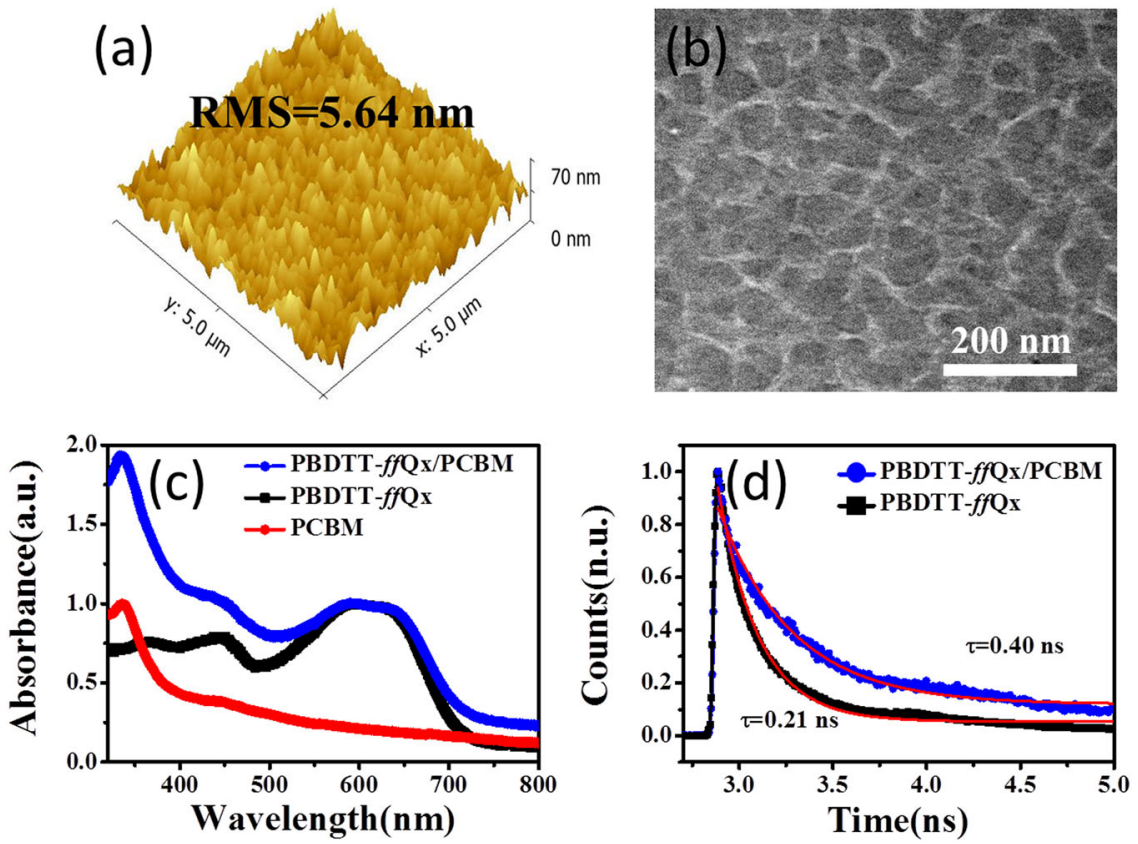

Fig. 2 a AFM and $\mathbf{b}$ TEM images of PBDTT-ffQx/PCBM BHJ thin film. c UV-vis absorption spectra of PBDTT-ffQx (black), PCBM (red), and PBDTT-

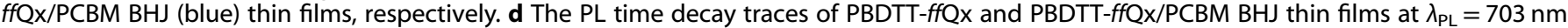
with the exponential fits $\left(y=y_{0}+A e^{-x / \tau}\right)$, of which the lifetime is 0.21 and $0.40 \mathrm{~ns}$, respectively
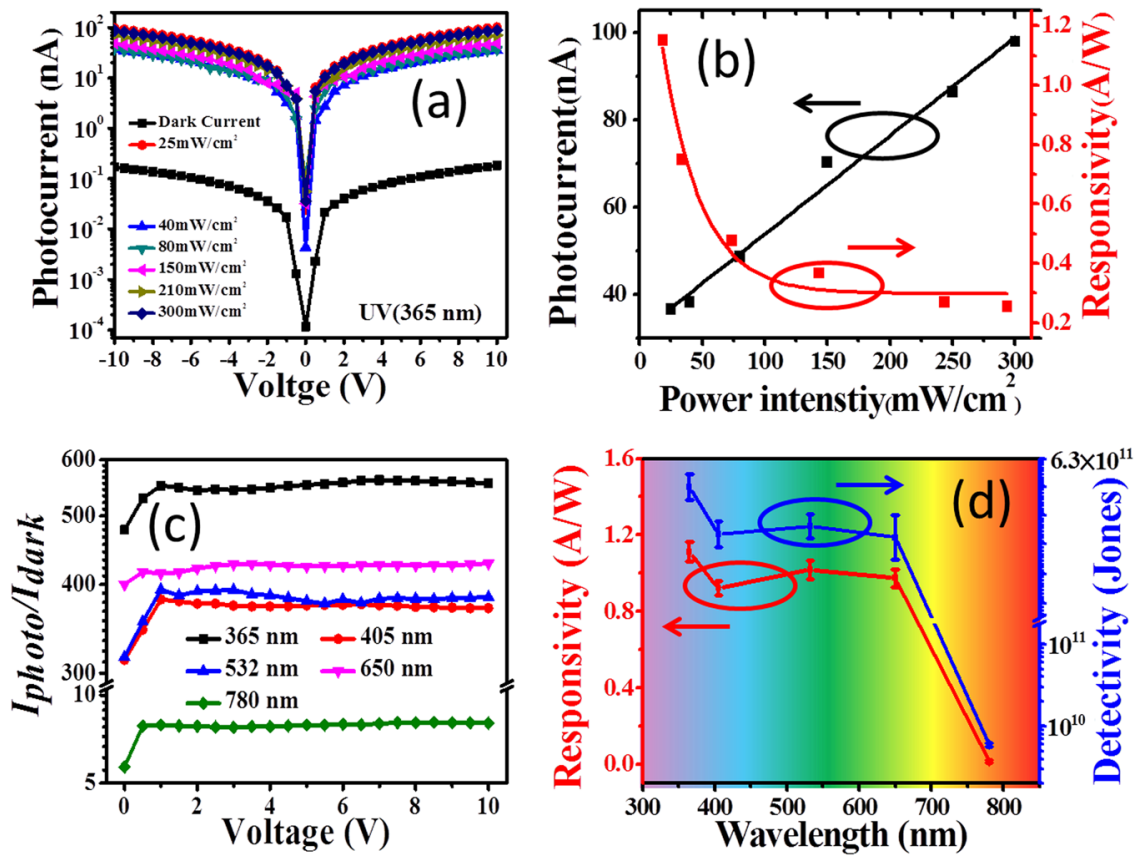

Fig. 3 a $I-V$ curves of PBDTT-ffQx/PCBM BHJ photodetector in dark and under $365 \mathrm{~nm}$ light illumination at the different light density. b Photocurrent and $R$ as a function of the light intensity at $10 \mathrm{~V} . \mathbf{c} I_{\text {photo }} / l_{\text {dark }}$ as a function of the bias voltage at the different wavelength. $\mathbf{d} R$ and $D^{*}$ versus the wavelength for the PBDTT-ffQx/PCBM BHJ photodetector. The data are the average value of five photodetector devices

PBDTT-ffQx/PCBM BHJ photodetector

The PBDTT-ffQx/PCBM BHJ thin film was used to fabricate the photodetector, and the photosensitivity could be confirmed by photocurrent measurements at the different power intensity, as shown in Fig. 3a and Figure S4. The photodetector shows strong intensity-dependent properties, i.e., the photocurrent increases with the light intensity linearly resulting from the number of photo-excited charge increases with the photon counts. The photocurrent comes from the transport of photo-generated carriers under the illumination. Under the illumination at the wavelength of $365 \mathrm{~nm}$, the current of the photodetector is enhanced dramatically from about 0.17 to $97.9 \mathrm{nA}$. Due to the generation of $\mathrm{e}-\mathrm{h}$ pairs through the absorption of incident photons, the PBDTT-ffQx/PCBM BHJ thin film becomes more 

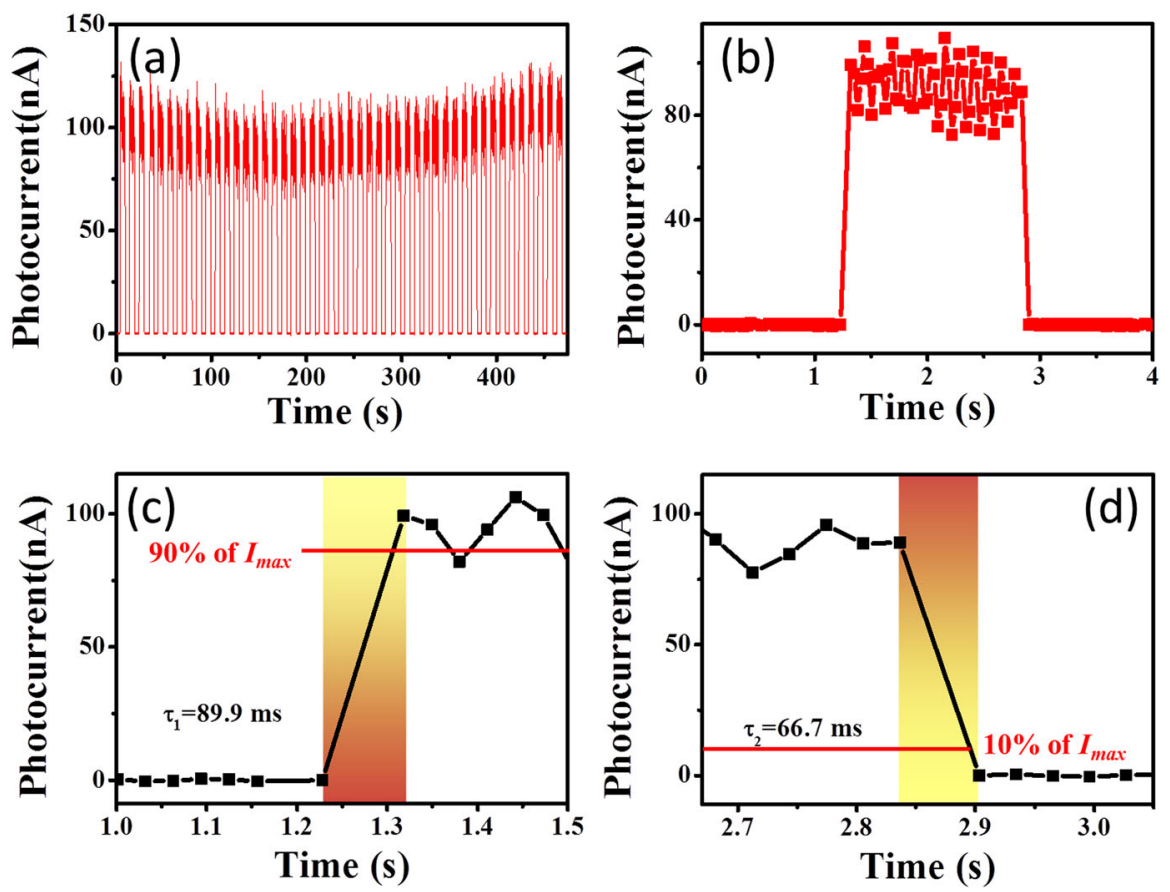

Fig. 4 a The reproducible on/off switching based on the PBDTT-ffQx/PCBM BHJ photodetector under $365 \mathrm{~nm}$ light illumination at $10 \mathrm{~V}$. b The photocurrent response is excited at $365 \mathrm{~nm}\left(300 \mathrm{~mW} / \mathrm{cm}^{2}\right)$. The magnified images of $\mathbf{c}$ rise time $\left(\tau_{1}\right)$ and decay time $\left(\tau_{2}\right)$, in which the $\tau_{1}$ is $89.9 \mathrm{~ms}$ and the $\tau_{2}$ is $66.7 \mathrm{~ms}$, respectively. $I_{\max }$ is the maximum value of photocurrent

conductive under the illumination. Especially, the devices have a photoresponse in a broad wavelength region from 365 to $808 \mathrm{~nm}$, which is consistent with the results of UV-Vis absorption spectra.

The $R$ is an important parameter to describe the photodetector performance, and it can be obtained by the equation:

$$
R=\frac{I_{\text {photo }}-I_{\text {dark }}}{P S},
$$

where $I_{\text {photo }}$ is the photocurrent, $I_{\text {dark }}$ is the dark current, $P$ is the incident light density, and $S$ is the effective illuminated area. ${ }^{27,28}$ The variations of photocurrent and $R$ with the light density are shown in Fig. 3b. The photocurrent of the photodetector increases with the power intensity, while the $R$ decreases dramatically and then remains at a value of about $0.3 \mathrm{~A} / \mathrm{W}$. Under $365 \mathrm{~nm}$ light illumination, the highest $R$ is up to $1.15 \mathrm{~A} / \mathrm{W}$. As the photodetector is illuminated under the low light power density, the $R$ values are very large, while it will be almost a constant value under the strong light, resulting from the dominated status of bimolecular recombination under strong light. 29,30

The values of $I_{\text {photo }} / I_{\text {dark }}$ as a function of voltage at the different wavelength are shown in Fig. 3c. Notably, the PBDTT-ffQx/PCBM $\mathrm{BHJ}$ photodetector exhibits a pronounced response in the UV and Vis region. The highest $I_{\text {photo }} / I_{\text {dark }}$ up to $5.61 \times 10^{2}$ is obtained at $365 \mathrm{~nm}$. Under Vis $(532 \mathrm{~nm})$ and NIR $(780 \mathrm{~nm})$ illumination, the values of $I_{\text {photo }} / I_{\text {dark }}$ are decreased to be about 394 and 8 , respectively. The wavelength dependence of $R$ and $D^{*}$ is plotted in Fig. $3 \mathrm{~d}$. The $R$ over $0.92 \mathrm{~A} / \mathrm{W}$ can be obtained in the wavelength range from 365 to $650 \mathrm{~nm}$, indicating that the PBDTT-ffQx/PCBM $\mathrm{BHJ}$ photodetector exhibits pronounced response from UV to the Vis light, which can be comparable to inorganic photodiodes and the fullerene based organic photodetectors. ${ }^{21}$ Interestingly, the best $R$ up to $1.15 \mathrm{~A} / \mathrm{W}$ can be achieved at $365 \mathrm{~nm}$, because the blending of PBDTT-ffQx and PCBM can enhance the light absorption coefficient in UV wavelength.
The $D^{*}$ is another important parameter for describing the detector performance and it can be calculated from the equation:

$$
D^{*}=\frac{R}{\left(2 q l_{\mathrm{d}} / A\right)^{1 / 2}},
$$

where $R$ is the responsivity, $A$ is the area of the detector, $q$ is the unit charge, and $I_{\mathrm{d}}$ is dark current. ${ }^{31}$ Owning to the suppressed dark current and enhanced responsivity of the PBDTT-ffQX/PCBM $\mathrm{BHJ}$ photodetector, the best value of $D^{*}$ is as high as $6.19 \times 10^{11}$ Jones under the illumination at the wavelength of $365 \mathrm{~nm}$. The values are $6.06 \times 10^{11}$ and $5.92 \times 10^{9}$ Jones under the illumination at the wavelength of 650 and $780 \mathrm{~nm}$, respectively.

The excellent repeatability and fast response time are the two important factors for the actual application of the photodetector. As shown in Fig. 4a, the reversible switching of the PBDTT-ffQx/ $\mathrm{PCBM} \mathrm{BHJ}$ photodetector is almost exactly reproducible for 50 on/ off cycles, revealing the good photoresponse stability. The photoresponse of typical single normalized cycle is shown in Fig. $4 \mathrm{~b}$. The rise time $\left(\tau_{1}\right)$ and fall time $\left(\tau_{2}\right)$ could be measured, which are defined as the time to transition between the minimum current to the $90 \%$ of the maximum value, and from the maximum value down to its $10 \%$, respectively. ${ }^{32-34}$ The magnified images of rise time and decay time are shown in Fig. $4 c$ and $d$, in which the $\tau_{1}$ is $88.9 \mathrm{~ms}$ and the $\tau_{2}$ is $66.7 \mathrm{~ms}$, respectively. The photoresponse is related to the extraction of the photo-generated charge carriers. Due to the rapid separation of photon-generated e- $h$ pairs at the interface and the high charge mobility, a fast response time of the PBDTT-ffQx/PCBM BHJ photodetector can be obtained.

To accurately evaluate the homogeneity of R2R micro-gravure printed PBDTT-ffQx/PCBM thin film, the large-scale PBDTT-ffQx/ PCBM BHJ photodetector array on flexible PET and paper were fabricated. The distribution diagram of the photocurrent and dark current are shown in Fig. 5a. The results were measured using 30 devices under $300 \mathrm{~mW} / \mathrm{cm}^{2}$ light irradiance at $365 \mathrm{~nm}$ and $10 \mathrm{~V}$ for a large scale, R2R micro-gravure printed PBDTT-ffQx/PCBM BHJ thin film. The photocurrents mainly distribute between 85 and $110 \mathrm{nA}$, while the dark current is between 0.16 and $0.19 \mathrm{nA}$. The 

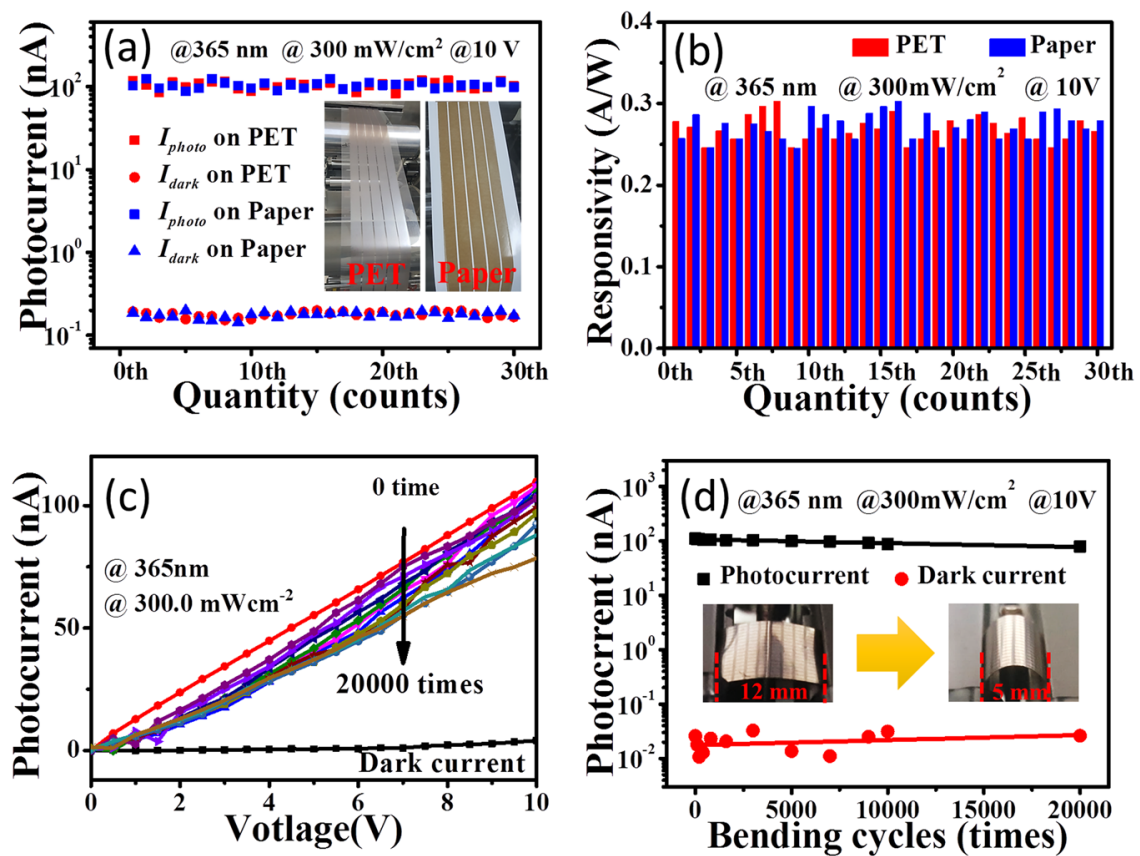

Fig. 5 a Distribution diagram of photocurrent and dark current of PBDTT-ffQx/PCBM BHJ photodetectors in a large-scale thin film on flexible PET and paper substrates. The insets are the photos of BHJ thin films on PET and paper substrates, respectively. (b) Statistical chart of $R$ values of photodetector array on PET and paper substrates. (c) I-V curves of PBDTT-ffQx/PCBM BHJ photodetector measuring under $365 \mathrm{~nm}$ illumination for bending from 0 to 20,000 cycles. d The change trends of photocurrent and dark current of the PBDTT-ffQx/PCBM BHJ photodetector with 20,000 bending cycles. The inset shows the bending process of PBDTT-ffQx/PCBM BHJ photodetector from the original state
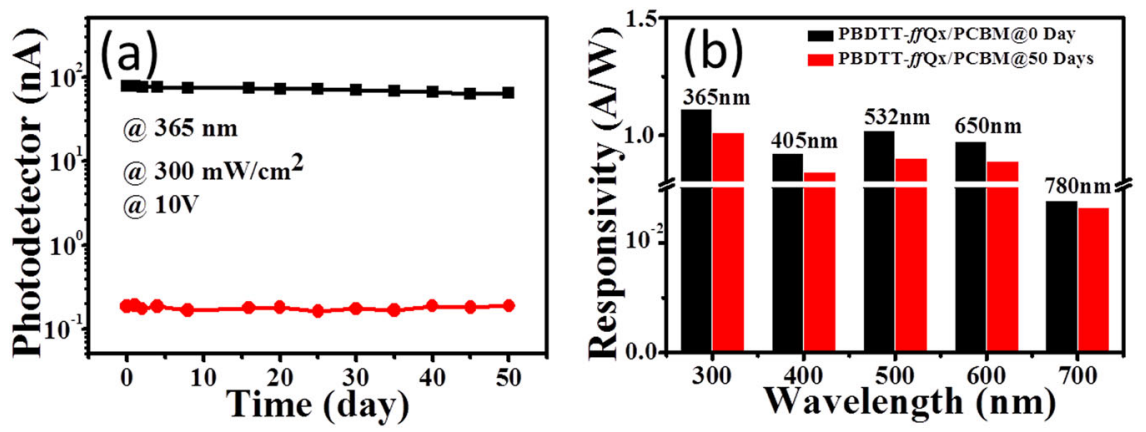

Fig. 6 a Photocurrent and dark current of PBDTT-ffQx/PCBM BHJ photodetector measured in ambient condition for exposing 50 days. $\mathbf{b}$ The $R$ values of the PBDTT-ffQx/PCBM BHJ photodetector in the UV-Vis regions in ambient condition at the original state and after exposing for 50 days

narrow distribution of photocurrent and dark current suggest that PBDTT-ffQx/PCBM BHJ thin film fabricated via R2R micro-gravure printing is very homogeneous. Furthermore, the $R$ values of photodetector array on PET and paper substrates are obtained as well, as shown in Fig. 5b. The average $R$ value for the photodetectors on the PET substrate is about $0.268 \mathrm{~A} / \mathrm{W}$ with a standard deviation of 0.015 . For the photodetectors on paper substrate, the average value and standard deviation are $0.272 \mathrm{~A} / \mathrm{W}$ and 0.016 , respectively. The results clearly manifests that the $R$ values fluctuate in a small scope, implying the good reliability and reproducibility for $\mathrm{R} 2 \mathrm{R}$ micro-gravure printed, large-area PBDTT$f f Q x / P C B M B H J$ thin film.

The flexibility of the R2R micro-gravure printed PBDTT-ffQx/ PCBM BHJ photodetector on PET substrates was measured and the results are shown in Fig. $5 c, d$. By controlling the distance between the adjacent stages, as shown in the inset in Fig. $5 d$, the bending radius of the PBDTT-ffQx/PCBM BHJ photodetector could be precisely controlled. The distance of adjacent stages is controlled from 12 to $5 \mathrm{~mm}$ via bending the flexible PBDTT-ffQx/ PCBM BHJ photodetector array followed by releasing it back to original state, of which the whole process is considered as one cycle. As shown in Fig. $5 c$, the $I-V$ curves of a flexible PBDTT-ffQx/ PCBM BHJ photodetector on a PET substrate was tested for bending from 0 to 20,000 cycles, of which the tested device is positioned at the center of the flexible photodetetor array. Owning to intrinsic flexible property of PBDTT-ffQx and PCBM semiconductor materials, the photocurrent changes little in the first 5000 cycles, just decreasing from 109 to $99 \mathrm{nA}$. When the bending cycles is increased to 10,000 cycles, the photocurrent only decreases to $88.0 \mathrm{nA}$, which is still more than $80 \%$ of the initial value. What is more, it still can remain over $70 \%$ of the initial value even after being bended for 20,000 cycles.

The stability is critical for practical application of photodetectors as well. The PBDTT-ffQx/PCBM BHJ photodetectors were stored in ambient conditions without any encapsulation (humidity $\sim 50 \%$, temperature $\sim 30^{\circ} \mathrm{C}$ ). As shown in Fig. 6 a, during the storage in 

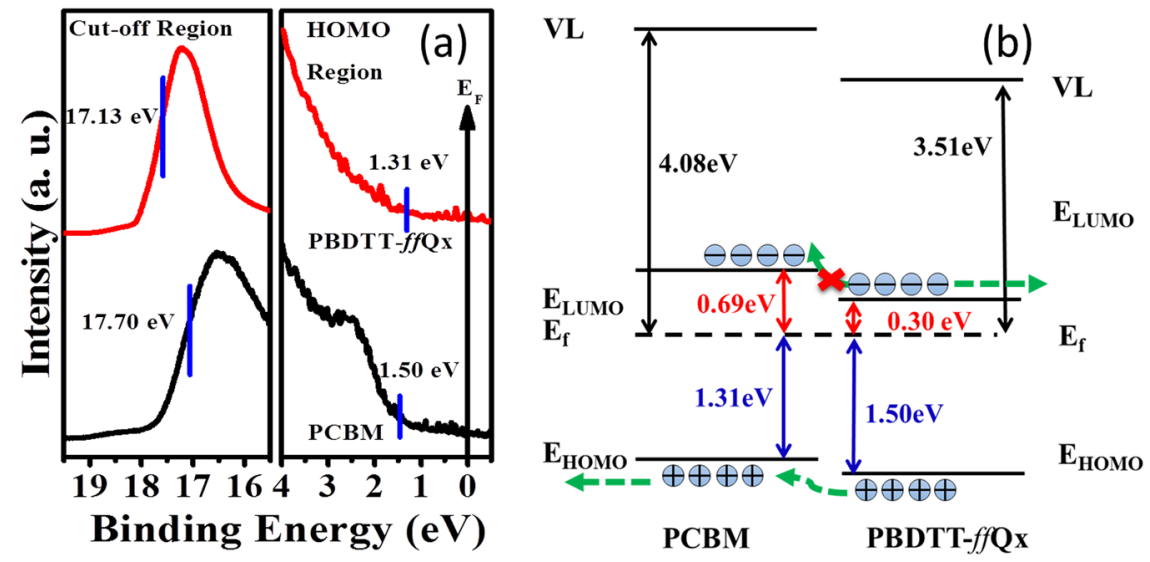

Fig. 7 a UPS spectra of cut-off region and HOMO region in PBDTT-ffQx and PCBM thin films. b Schematic energy levels of the PBDTT-ffQx and PCBM thin films

ambient condition for 50 days, the photocurrent just decreased from 78.5 to $63.5 \mathrm{nA}$, i.e., about $20 \%$ degradation. Meanwhile, the dark currents are almost the same, proving that the PBDTT-ffQx/ PCBM BHJ photodetector exhibits excellent stability. The $R$ values for 50 days storage in the UV-Vis regions is shown in Fig. 6b. The $R$ values could maintain over $80 \%$ performance from UV to Vis regions. The excellent stability could be attributed to the intrinsically stable PBDTT-ffQx. Meanwhile, the stability could be dramatically improved via blending polymer semiconductors with fullerene materials, in which the formation of charge transfer complexes could be avoided. ${ }^{35-37}$ As compared to recent progresses on organic $\mathrm{BHJ}$ photodetectors fabricated via spincoating or thermal evaporation, the R2R micro-gravure printed, large-area and flexible PBDTT-ffQx/PCBM BHJ photodetector has significant advantages on $R$ and stability, and the results are summarized in the Table S1.

Mechanism analysis of PBDTT-ffQx/PCBM BHJ thin film In order to analyze the charge transport mechanism between PBDTT-ffQx and PCBM, ultraviolet photoemission spectroscopy (UPS) was used to measure the interface electronic structure. The work function $\left(W_{\mathrm{F}}\right)$ of PBDTT-ffQx and PCBM thin films were measured. It is the difference between the cut-off region and the Fermi energy $\left(E_{\mathrm{f}}\right)$ of the system $(21.21 \mathrm{eV})$. As shown in Fig. 7a, the binding energy is about $3.51 \mathrm{eV}$ for the PBDTT-ffQx thin film and $4.08 \mathrm{eV}$ for the PCBM thin film. The band gaps of PBDTT-ffQx and $\mathrm{PCBM}$ are 1.80 and $2.0 \mathrm{eV}$, respectively. ${ }^{20}$ The schematic energy levels of the PBDTT-ffQx and PCBM is shown in Fig. 7b. The highest occupied molecular orbital (HOMO) of thin films could be acquired directly from Fig. 7a. The gap between the HOMO and the $E_{\mathrm{f}}$ for the PBDTT-ffQx thin film is $1.50 \mathrm{eV}$, which is larger than the value $(1.31 \mathrm{eV})$ of the PCBM thin film, indicating that there is an effective hole transport path from the PBDTT-ffQx to the PCBM. The difference between the lowest unoccupied molecular orbital and the $E_{\mathrm{f}}$ for the PBDTT-ffQx thin film is $0.30 \mathrm{eV}$, which is much lower than the value of $0.69 \mathrm{eV}$ for the PCBM thin film, indicating that the electron transport from the PBDTT-ffQx to the PCBM would be prevented. Under the light illumination, the mainly photogenerated e-h pairs could be formed in PBDTT-ffQx and separated at the PBDTT-ffQx/PCBM interface. Owning to the ability of transporting hole, ${ }^{24,25}$ the photo-generated holes can be effectively extracted from the PBDTT-ffQx and transport in the PCBM to the electrode. Meantime, the photo-generated electrons can transport in the PBDTT-ffQx polymer network as well, which are linked by the D-A interface of polymer chains. Because of the energy level matching between the PBDTT-ffQx and the PCBM, the photo-generated e-h could be separated effectively, which would lead to a high photosensitivity for the PBDTT-ffQx/PCBM BHJ photodetector.

For further analyzing the molecular structure of $\mathrm{BHJ}$ thin film, Xray photoelectron spectroscopy (XPS) was used, and the results are shown in Figure S5. As shown in molecular structure of PBDTT$f f Q x$ (Fig. 1), each PBDTT-ffQx unit has four $F$ atoms and six $S$ atoms, while the PCBM does not have these atoms, indicating that $S$ and $F$ are the characteristic elements in PBDTT-ffQx. The results suggest that both PBDTT-ffQx and PBDTT-ffQx/PCBM BHJ thin films have the same binding energy for the $F 1 s$ and $S 2 p$, demonstrating that no chemical bond forms between the PBDTT$f f Q x$ and the PCBM. In addition, the binding energy of $N$ element is the same as well. XPS results prove the formation of $\mathrm{BHJ}$ thin film via blending the PBDTT-ffQx and the PCBM, while not the formation of chemical bonds.

In summary, the large area, flexible organic photodetector based on PBDTT-ffQx/PCBM BHJ were fabricated by the largescale, R2R micro-gravure printing technique on PET and paper substrates. The PBDTT-ffQx/PCBM BHJ photodetector showed a broadband wavelength response from UV to Vis, a high $D^{*}$ value up to $6.19 \times 10^{11}$ Jones, and an excellent $I_{\text {photo }} / I_{\text {dark }}$ as high as $5.6 \times 10^{2}$. The PBDTT-ffQx/PCBM BHJ photodetector was very flexible and stable. Being bended for 10,000 cycles or exposed to ambient condition for 50 days without any encapsulation, it could maintain the $80 \%$ of original performance parameters. More importantly, the large-area PBDTT-ffQx/PCBM BHJ thin film fabricated by R2R micro-gravure printing is very homogeneous, and the photodetector devices showed excellent reliability and reproducibility. It is believed that the flexible and stable PBDTT$f f Q x / P C B M B H J$ photodetector fabricated via the large-scale, highoutput R2R micro-gravure printing technique would be potentially matched with low-cost portable and wearable optoelectronic applications.

\section{METHODS}

\section{Device fabrication}

PBDTT-ffQx was synthesized via Stille coupling reaction in toluene using Pd $\left(\mathrm{PPh}_{3}\right)_{4}$ (Acros Organics) as the catalyst. The polymer was purified by continuous Soxhlet extraction using methanol, hexane, and chloroform. The chloroform fraction was recovered and the solution was filtered through a silica gel column. The polymer was precipitated into methanol and collected via filtration. The previous work demonstrated the detailed experimental procedures for the synthesis of the monomers and copolymer of PBDTT-ffQx. ${ }^{20}$ Then, the precursor solution was prepared by mixing PBDTT-ffQx and PCBM (Dye Source, American) at a weight ratio of 1:1 in anhydrous chlorobenzene (CB, J\&K Seal) with the concentration of $20 \mathrm{mg} / \mathrm{ml}$. Finally, a $3 \%$ 1,8-diiodooctane (TCl Chemical Co.) volume 
amount was added in precursor solution, and stirred for $3 \mathrm{~h}$ for the subsequent printing fabrication.

The R2R micro-gravure printing was used to produce large-scale PBDTTffQx/PCBM BHJ thin film using a self-developed R2R printing machine. A patterned micro-gravure roller was engraved with a line density of 80 lines per $\mathrm{cm}$, a spiral rib angle of $45^{\circ}$, and a depth of $45 \mu \mathrm{m} .{ }^{17,38}$ The formation process and the thickness of printed PBDTT-ffQx/PCBM BHJ thin film could be controlled via micro-gravure printing roller speed and web speed. Finally, R2R micro-gravure printed PBDTT-ffQx/PCBM BHJ thin film was dried in air condition naturally (temperature: $\sim 30^{\circ} \mathrm{C}$, humidity: $\sim 40 \%$ ). The gold and silver $(\mathrm{Au} / \mathrm{Ag})$ electrodes $(80 / 220 \mathrm{~nm})$ were thermally evaporated on the top of printed PBDTT-ffQx/PCBM BHJ thin film through a carbon fiber barred shadow mask with a channel width of $8 \mu \mathrm{m}$ and channel length of $200 \mu \mathrm{m}$, respectively.

\section{Measurements}

All the samples were characterized in ambient conditions. The surface morphology of thin films was examined by using atomic force microscopy (Agilent Technologies 5500 AFM/SPM System, USA) and a transmission electron microscope (TEM, JEM-2100F, Japan). The absorption spectra and cross-section images of thin films were obtained by ultraviolet-visible spectrophotometer (UV-vis; Puxi, T9, China) and SEM (FEl Helios Nanolab $600 \mathrm{i}$, USA). The spectroscopies of thin films were measured by an XPS system (ESCALAB 250Xi; ThermoFisher-VG Scientific) and UPS in a ultrahigh vacuum system, of which the pressure of analysis chamber is superior to $5 \times 10^{-10} \mathrm{mbar}$. The steady-state $\mathrm{PL}$ spectra were achieved using an intensified charge coupled device detector (DH334T-18U-03). The PL lifetime were measured using time-correlated single photon counting (MS3504I) measurements. The photodetector performance was measured using a probe station connected to a Keithley 4200 SCS semiconductor parameter analyzer under ambient conditions. The light power intensity was adjusted by insetting attenuation slices between the light source and the samples.

\section{Data availability}

The data that support the findings of this study are available from the authors on reasonable request. The authors declare that the data supporting the findings of this study are available within the article and its Supplementary Information file.

\section{ACKNOWLEDGEMENTS}

This work was supported by the National Natural Science Foundation of China (51673214), the National Key Research and Development Program of China (2017YFA0206600), the Hunan Provincial Natural Science Foundation of China (2015JJ1015), and the Project of Innovation-driven Plan in Central South University (2015CXS036). S.T. thanks the Doctoral Innovation Fund of Central South University (2017ZZTS063)

\section{AUTHOR CONTRIBUTIONS}

S. T. and J. Y. conceived the idea. S. T. performed the fabrication and characterization on photodetectors. S. T., J. S., and J. Y. analyzed all the performance of printed films and photodetectors, as well as wrote the manuscript. J. Y. and Y. Z. designed and synthesized PBDTT-ffQx. C. W. and H. X. developed and optimized the BHJ film growth. C. Z. conducted and analyzed the SEM measurements. J. S., S. X., and J. H. conducted and analyzed the PL lifetime measurements. B. L., H. X., and Y. G. conducted and analyzed the UPS and XPS measurements. J. Y. directed the overall research plan. All the authors read and commented on the manuscript.

\section{ADDITIONAL INFORMATION}

Supplementary information accompanies the paper on the npj Flexible Electronics website (https://doi.org/10.1038/s41528-017-0020-y).

Competing interests: The authors declare no competing financial interests.

Publisher's note: Springer Nature remains neutral with regard to jurisdictional claims in published maps and institutional affiliations.

\section{REFERENCES}

1. Tong, S. et al. High-performance broadband perovskite photodetectors based on $\mathrm{CH}_{3} \mathrm{NH}_{3} \mathrm{Pbl}_{3} / \mathrm{C} 8 \mathrm{BTBTT}$ heterojunction. Adv. Electron. Mater. 3, 1700058 (2017).

2. Gao, L. et al. Broadband, sensitive and spectrally distinctive $\mathrm{SnS}_{2}$ nanosheet/PbS colloidal quantum dot hybrid photodetector. Light. Sci. Appl. 5, 1-8 (2016).

3. Gou, G. et al. High-performance ultraviolet photodetectors based on $\mathrm{CdS} / \mathrm{CdS}$ : $\mathrm{SnS}_{2}$ superlattice nanowires. Nanoscale 8, 14580-14586 (2016).

4. Qian, C., Kong, L., Yang, J., Gao, Y. \& Sun, J. Multi-gate organic neuron transistors for spatiotemporal information processing. Appl. Phys. Lett. 110, 083302 (2017).

5. Pyo, S., Kim, W., Jung, H., Choi, J. \& Kim, J. Heterogeneous integration of carbonnanotube-graphene for high-performance, flexible, and transparent photodetectors. Small 13, 1700918 (2017).

6. Zhang, K. et al. A flexible $\mathrm{p}-\mathrm{CuO} / \mathrm{n}-\mathrm{MoS}_{2}$ heterojunction photodetector with enhanced photoresponse by the piezo-phototronic effect. Mater. Horiz. 4, 274-280 (2017).

7. Soares, R. R. G. et al. A point-of-use microfluidic device with integrated photodetector array for immunoassay multiplexing: detection of a panel of mycotoxins in multiple samples. Biosens. Bioelectron. 87, 823-831 (2016).

8. Jo, S. H. et al. Broad detection range rhenium diselenide photodetector enhanced by (3-aminopropyl)triethoxysilane and triphenylphosphine treatment. Adv. Mater. 28, 6711-6718 (2016).

9. Yuan, J. et al. Tetrafluoroquinoxaline based polymers for non-fullerene polymer solar cells with efficiency over 9\%. Nano Energy 30, 312-320 (2016).

10. Yang, J., Yan, D. \& Jones, T. S. Molecular template growth and its applications in organic electronics and optoelectronics. Chem. Rev. 115, 5570-5603 (2015).

11. Sun, Y. et al. Efficient, air-stable bulk heterojunction polymer solar cells using $\mathrm{MoO}_{\mathrm{x}}$ as the anode interfacial layer. Adv. Mater. 23, 2226-2230 (2011).

12. Chu, T. et al. High-efficiency inverted solar cells based on a low bandgap polymer with excellent air stability. Sol. Energ. Mat. Sol. C. 96, 155-159 (2012).

13. Yuan, J. et al. Development of quinoxaline based polymers for photovoltaic applications. J. Mater. Chem. C. 5, 1858-1879 (2017)

14. Shen, L. et al. Integration of perovskite and polymer photoactive layers to produce ultrafast response, ultravioletto-near-infrared, sensitive photodetectors. Mater. Horiz. 4, 242-248 (2017).

15. $\mathrm{Hu}, \mathrm{X}$. et al. Roll-to-Roll production of graphene hybrid electrodes for high-efficiency, flexible organic photoelectronics. Adv. Mater. Interfaces 2, 1500445 (2015).

16. Park, J., Lee, J., Noh, Y., Shin, K. \& Lee, D. Flexible ultraviolet photodetectors with $\mathrm{ZnO}$ nanowire networks fabricated by large area controlled roll-to-roll processing J. Mater. Chem. C. 4, 7948-7958 (2016).

17. Zhang, C. et al. Roll-to-roll micro-gravure printed large-area zinc oxide thin film as the electron transport layer for solution-processed polymer solar cells. Org. Electron. 45, 190-197 (2017).

18. Yang, C. et al. Textured V-Pit green light emitting diode as a wavelength-selective photodetector for fast phosphor-based white light modulation. ACS Photonics 4 443-448 (2017).

19. Fang, J. et al. Enhanced graphene photodetector with fractal metasurface. Nano Lett. 17, 57-62 (2017).

20. Yuan, J. et al. A simple strategy to the side chain functionalization on the quinoxaline unit for efficient polymer solar cells. Chem. Commun. 52, 6881-6884 (2016).

21. Wang, T. et al. High sensitivity, fast response and low operating voltage organic photodetectors by incorporating a water/alcohol soluble conjugated polymer anode buffer layer. RSC Adv. 7, 1743-1748 (2017).

22. Pace, G. et al. All-organic and fully-printed semitransparent photodetectors based on narrow bandgap conjugated molecules. Adv. Mater. 26, 6773-6777 (2014).

23. Shi, D. et al. Low trap-state density and long carrier diffusion in organolead trihalide perovskite single crystals. Science 347, 519-522 (2015).

24. Yang, J. L., Sullivan, P., Schumann, S., Hancox, I. \& Jones, T. S. Organic photovoltaic cells based on unconventional electron donor fullerene and electron acceptor copper hexadecafluorophthalocyanine. Appl. Phys. Lett. 100, 023307 (2012).

25. Song, Q., Yang, H., Gan, Y., Gong, C. \& Ming, L. Evidence of arvesting electricity by exciton recombination in an n-n type solar cell. J. Am. Chem. Soc. 132, 4554-4555 (2010).

26. Wang, $\mathrm{C}$. et al. Air-induced high-quality $\mathrm{CH}_{3} \mathrm{NH}_{3} \mathrm{Pbl}_{3}$ thin film for efficient planar heterojunction perovskite solar cells. J. Phys. Chem. C. 121, 6575-6580 (2017).

27. Li, S. et al. High-performance formamidinium-based perovskite photodetectors fabricated via doctor-blading deposition in ambient condition. Org. Electron. 47, 102-107 (2017).

28. Velusamy, D. B. et al. 2D organic-inorganic hybrid thin films for flexible UV-Visible photodetectors. Adv. Funct. Mater. 27, 1605554 (2017).

29. Binet, F., Duboz, J. Y., Rosencher, E., Scholz, F. \& Härle, V. Mechanisms of recombination in GaN photodetectors. Appl. Phys. Lett. 69, 1202-1204 (1996). 
30. Stevens, K. S., Kinniburgh, M. \& Beresford, R. Photoconductive ultraviolet sensor using Mg-doped GaN on Si(111). Appl. Phys. Lett. 66, 3518-3520 (1995).

31. $\mathrm{Yu}, \mathrm{S}$. H. et al. Photoconductive ultraviolet sensor using Mg-doped $\mathrm{GaN}$ on $\mathrm{Si}$ (111). Appl. Phys. Lett. 66, 3518-3520 (1995).

32. Mehew, J. D. et al. Fast and highly sensitive ionic-polymer-gated $\mathrm{WS}_{2}$-graphene photodetectors. Adv. Mater. 29, 1700222 (2017)

33. Saidaminov, M. I. et al. Planar-integrated single-crystalline perovskite photodetectors. Nat. Commun. 6, 8724 (2015).

34. Heo, K. et al. Aligned networks of cadmium sulfidenanowires for highly flexible photodetectors with improved photoconductive responses. J. Mater. Chem. 22, 2173-2179 (2012)

35. Kumar, A. et al. Origin of radiation-induced degradation in polymer solar cells. Adv. Funct. Mater. 20, 2729-2736 (2010).

36. Yang, J. et al. Solution stability of active materials for organic photovoltaics. Sol. Energy 113, 181-188 (2015).

37. Reese, M. O. et al. Photoinduced degradation of polymer and polymer-fullerene active layers: experiment and theory. Adv. Funct. Mater. 20, 3476-3483 (2010).
38. Hu, Q. et al. Large-area perovskite nanowire arrays fabricated by large-scale rollto-roll micro-gravure printing and doctor blading. Nanoscale 8, 5350-5357 (2016).

cc (i) Open Access This article is licensed under a Creative Commons Attribution 4.0 International License, which permits use, sharing, adaptation, distribution and reproduction in any medium or format, as long as you give appropriate credit to the original author(s) and the source, provide a link to the Creative Commons license, and indicate if changes were made. The images or other third party material in this article are included in the article's Creative Commons license, unless indicated otherwise in a credit line to the material. If material is not included in the article's Creative Commons license and your intended use is not permitted by statutory regulation or exceeds the permitted use, you will need to obtain permission directly from the copyright holder. To view a copy of this license, visit http://creativecommons. org/licenses/by/4.0/.

(c) The Author(s) 2018 